\title{
Análise facial frontal em repouso e durante o sorriso em fotografias padronizadas. Parte II: Avaliação durante o sorriso*
}

\author{
Vera Lúcia COLOMBO**, Alexandre MORO***, Régis $\mathrm{RECH}^{\star *}$, Janaína VERONA****, \\ Gilce C. Alves da COSTA ${ }^{\star \star \star \star \star}$
}

\section{Resumo}

Este artigo compreende a segunda parte da análise facial frontal em fotografias padronizadas. Fizeram parte da amostra estudada 40 mulheres, com idade média de 22 anos, leucodermas, com faces agradáveis, Classe I de Angle, sem história prévia de tratamento ortodôntico e cirurgia plástica na face. Foram obtidas fotografias faciais frontais (10x15 cm coloridas), padronizadas. Duas fotografias foram obtidas, no sorriso máximo e durante o repouso. Sobre as fotografias, foram realizadas medidas lineares, angulares e proporcionais. As medidas foram avaliadas por meio de teste de normalidade, estatística descritiva e desvio padrão do erro. Os resultados mostraram que algumas das medidas utilizadas neste trabalho se assemelham às encontradas na literatura e outras diferem muito. E a partir desse estudo propõem-se uma análise facial frontal para utilização rotineira no diagnóstico e planejamento do tratamento ortodôntico.

Palavras-chave: Análise facial frontal. Ortodontia. Estética facial. Sorriso.

\section{INTRODUÇÃO}

Muito tem sido escrito $4,5,9,10,12,18,26,33,40$ sobre o conceito de beleza e estética facial, mas sua discussão dentro da Ortodontia é um tanto limitada, particularmente com relação à quantificação dos tecidos moles faciais de contorno e, também, pouca alusão tem sido feita à análise do sorriso, apesar de ser um dos principais motivos para a procura do tratamento ortodôntico. Para Jansen ${ }^{21}$ um sorriso bem equilibrado é um dos mais importantes objetivos do tratamento ortodôntico.

A estética tem se tornado cada vez mais importante na Odontologia sendo sinônimo de uma aparência natural e harmoniosa. Para Tjan e Miller $^{39}$ um sorriso atrativo e agradável acentua a aceitação do indivíduo na sociedade, pela melhora na impressão inicial do relacionamento interpessoal. O sorriso é uma das mais importantes expressões faciais e é essencial na demonstração de amizade, concordância e apreciação.

* Resumo da Monografia apresentada ao Curso de Especialização em Ortodontia e Ortopedia Facial da EAP-ABO/PR, como requisito para obtenção do título de Especialista.

** Especialista em Ortodontia e Ortopedia Facial pela EAP-ABO/PR

*** Mestre e Doutor em Ortodontia, professor adjunto do Departamento de Anatomia da UFPR, professor dos Cursos de Especialização em Ortodontia e Ortopedia Facial da EAP-ABO/PR e UFPR, Orientador do trabalho.

$\star \star \star \star$ Aluna do Curso de Especialização em Ortodontia e Ortopedia Facial da EAP-ABO/PR

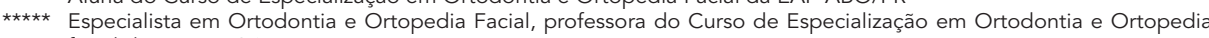
facial da EAP-ABO/PR. 
Quando as pessoas sorriem, é o relacionamento dos dentes com os lábios e posicionamento dos mesmos que determina o grau de atratividade. Os lábios geralmente são analisados no plano sagital, na posição estática, e pouca referência da linha estética do sorriso tem sido feita. O sorriso é um dos meios mais efetivos pelo qual as pessoas demonstram sua emoção. Um sorriso atraente e equilibrado pode ser o mais valioso recurso pessoal.

O sorriso atrativo é um complemento da beleza facial. Um sorriso simétrico, com dentes bem posicionados e alinhados no arco, uma exposição adequada dos dentes anteriores superiores, proporciona uma beleza ideal. Muitas pessoas, consideradas belas, quando sorriem apresentam um sorriso assimétrico ou um sorriso gengival, perdendo o encanto inicial. Hulsey ${ }^{20}$ observou que o sorriso é um dos meios mais eficazes pelo qual as pessoas transmitem suas emoções.

A análise da face durante o sorriso, antes do início do tratamento, foi ressaltada por alguns autores ${ }^{13,21,24,25}$. Na literatura, encontramos alguns estudos sobre o sorriso $1,17,20,30,36,37,38,39,41$. A análise facial frontal, durante o sorriso, permite avaliar o alinhamento das linhas médias dentária e facial, a inclinação do sorriso, o centro do sorriso, o tipo de musculatura predominante, a curvatura incisal, o corredor bucal e a exposição gengival, os quais determinam o grau de atratividade de um sorriso.

A linha do sorriso é muito importante para a estética facial ${ }^{16,23}$. Uma linha do sorriso alta (sorriso gengival) é considerada antiestética para algumas pessoas, por isso alguns autores preconizam técnicas para correção do sorriso gengival ${ }^{11,22,27}$.

Rigsbee III, Sperry e Begole ${ }^{36}$, em seus estudos concluíram que mudanças reproduzíveis dos lábios, nariz e mento, durante o sorriso, podem ser documentadas, e também que a fotografia do sorriso, feita com uma técnica padronizada, parece ser um auxílio-diagnóstico de reprodutibilidade segura.

Este trabalho se propôs a elucidar as seguintes questões: a) quais são os padrões médios de normalidade para as variáveis da análise da fotografia frontal durante o sorriso da amostra estudada?

b) as medidas obtidas nesta amostra assemelham-se às medidas encontradas na literatura?

c) qual a proporção existente entre algumas das medidas lineares da fotografia frontal, em repouso e durante o sorriso na amostra estudada?

d) qual o tipo de sorriso e curvatura incisal predominantes na amostra estudada?

e) as "Proporções Divinas" do sorriso propostas por Ricketts ${ }^{35}$ são válidas para a amostra estudada?

f) do ponto de vista da repetibilidade, as variáveis são confiáveis?

\section{MATERIAL E MÉTODO Material}

Fizeram parte da amostra estudada 40 indivíduos do gênero feminino, leucodermas, com idade entre 18 e 28 anos (média de 22 anos). Na obtenção da fotografia, foi utilizada uma máquina fotográfica Canon EOS Rebel X, lente macro 100/35 $\mathrm{mm}$ Canon, flash circular Canon, tripé Zenit TZ40, filme Kodak, colorido, ASA 100/35 mm, um espelho, um fio azul com um peso na extremidade (utilizado como referência vertical) e 80 fotografias coloridas, tamanho $10 \times 15 \mathrm{~cm}$.

\section{Método}

Fotografia

De todos os indivíduos foram realizadas fotografias frontais da face. Cada indivíduo foi fotografado sentado, olhando diretamente para seus olhos refletidos em um espelho colocado à sua frente ${ }^{14}$. Cada sujeito foi instruído a manter uma postura ereta, "natural e normal", com ambos os braços livres ao lado do tronco. Essa posição corresponde à "posição natural de cabeça" de Broca ${ }^{6,8,11,14,15,28}$. $\mathrm{Na}$ fotografia da face, durante o sorriso máximo (máxima contração muscular), as arcadas estavam em máxima intercuspidação habitual. Para obter-se uma referência vertical verdadeira, foi usado um 
fio preso ao teto, caindo ao lado da cabeça e com um peso em sua extremidade ${ }^{6}$ visível na fotografia.

A distância focal utilizada foi de um metro (dada pela regulagem da lente macro 100/35 $\mathrm{mm}$ ), a velocidade de 60 e a abertura da lente de 11. Para permitir uma correlação entre as medidas reais e as medidas obtidas nas fotografias, em 10 indivíduos foram marcados dois pontos na testa com uma distância de $1 \mathrm{~cm}$ entre eles. Também foi fotografada uma régua, nos mesmos padrões da fotografia da face. A medida de $0,55 \mathrm{~mm}$ da fotografia corresponde aproximadamente a $1 \mathrm{~cm}$ do tamanho real $\mathrm{da}$ face, isto é, a fotografia corresponde a $55 \%$ do tamanho real. A distorção é dada em números aproximados, já que, na fotografia, tem-se uma visão bidimensional da estrutura tridimensional da face.

Todos os filmes fotográficos foram revelados num mesmo laboratório Kodak, padrão QLab (padrão de qualidade internacional Kodak).

\section{SELEÇÃO DA AMOSTRA}

A amostra foi obtida nos estados do sul do Brasil e uma banca composta por professores e alunos do curso de pós-graduação em Ortodontia da ABO-PR selecionou 40 faces de indivíduos do gênero feminino, brancos, filhas de pais brasileiros de origem européia (italianos, alemães, portugueses, holandeses e espanhóis), com idade entre 18 e 28 anos, a partir de uma amostra prévia de 83 indivíduos. Esses indivíduos apresentavam os seguintes requisitos:

a) possuíam uma oclusão de Classe I (aceitou-se um apinhamento mínimo ou pequenos diastemas que não justificassem um tratamento ortodôntico);

b) possuir uma face agradável;

c) não ter sido submetida a tratamento ortodôntico;

d) não ter se submetido a nenhum tipo de cirurgia plástica facial.

\section{TRAÇADO FOTOMÉTRICO Desenho anatômico}

O desenho anatômico da face durante o sorriso foi realizado de forma que se permitisse o traçado dos pontos e linhas que seriam utilizados.

Pontos, linhas e medidas fotométricas

Os pontos fotométricos (Fig. 1) utilizados, além dos já descritos no primeiro artigo publicado ${ }^{7}$, foram:

BiLs - ponto médio da borda inferior do lábio superior.

BsLi - ponto médio da borda superior do lábio inferior.

ICS - borda inferior dos incisivos centrais superiores, no ponto médio entre os incisivos centrais direito e esquerdo.

CS - ponta da cúspide do canino superior direito (CSd) e esquerdo (Cse).

dCS - ponto mais distal na região média da coroa do canino superior direito

(dCSd) e esquerdo (dCSe).

Ces - Centro do sorriso - É traçada uma linha de referência que vai do canto direito ao canto esquerdo da boca. A partir desta linha, constrói-se duas linhas paralelas a esta e tangentes aos lábios superior e inferior, respectivamente. Traça-se, então, duas linhas perpendiculares à linha de referência, passando pelos cantos direito e esquerdo da boca, obtendo-se assim um retângulo. Ao traçarem-se duas linhas transversais neste retângulo, tem-se um ponto onde elas se cruzam. Este ponto é denominado de centro do sorriso ${ }^{29}$.

Linhas fotométricas

Linha interexocanto - Linha horizontal que se estende do exocanto direito (Exd) ao exocanto esquerdo (Exe).

Linha média facial - Linha traçada verticalmente passando pelo ponto násio (N') e o filtro inferior $(\mathrm{F})$.

Linha da comissura - Linha horizontal se estende do ângulo da boca no lado direito (Abd) 
ao ângulo da boca no lado esquerdo (Abe ${ }^{29}$.

Linha média dentária superior - Linha traçada perpendicularmente à linha da comissura, passando entre as coroas dos incisivos centrais superiores direito e esquerdo.

Medidas fotométricas lineares (Fig. 2, 3, 4)

Extensão do vermelhão do lábio superior (LsBiLs) - Distância linear do ponto médio da junção cutânea com o vermelhão do lábio superior (Ls) à borda inferior do lábio superior (BiLs).

Exposição dos incisivos centrais superiores (BiLs-ICS) - Distância linear da borda inferior do lábio superior (BiLs) à borda inferior dos incisivos centrais superiores, no ponto médio entre os incisivos centrais direito e esquerdo (ICS).

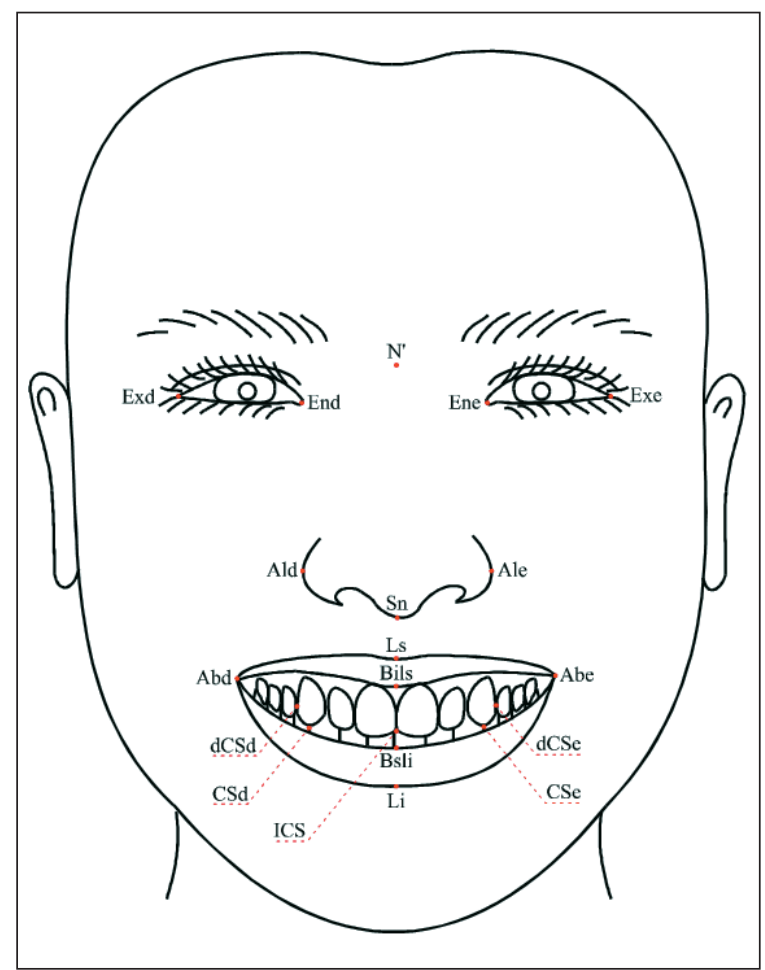

FIGURA 1 - Pontos fotométricos.

LEGENDA: N' - násio, Abd - ângulo da boca direito, Exd - exocanto direito, Abe - ângulo da boca esquerdo, Exe - exocanto esquerdo, Li - lábio inferior, End - endocanto direito, Bils - borda inferior do lábio superior, Ene - endocanto esquerdo, Bsli - borda superior do lábio inferior, Ald - alar direito, ICS - incisivo central superior, Ale - alar esquerdo, CSd - canino superior direito, $\mathbf{S n}$ - subnasal, CSe - canino superior esquerdo, F - filtro inferior *, dCSd - distal de canino superior direito, Ls - lábio superior, dCSe - distal de canino superior esquerdo, * F- (filtro inferior) coincidente com o ponto Ls (lábio superior).
Espaço interlabial (BiLs-BsLi) - Distância linear da borda inferior do lábio superior (BiLs) à borda superior do lábio inferior (BsLi).

Extensão do vermelhão do lábio inferior (BsLi-Li) - Distância linear da borda superior do lábio inferior (BsLi) ao ponto médio da junção cutânea com o vermelhão do lábio inferior (Li).

Altura do sorriso ( Ls-Li) - Distância linear do ponto médio da junção cutânea com o vermelhão do lábio superior (Ls) ao ponto médio da junção cutânea com o vermelhão do lábio inferior (Li).

Inclinação da linha da comissura - É a diferença, em milímetros, entre a distância da linha da comissura à linha interexocanto na altura do ângulo da boca direito (Abd) e a distância da linha da comissura à linha interexocanto na altura do ângulo da boca esquerdo (Abe).

Largura do nariz (Ald-Ale) - Distância linear do alar direito (Ald) ao alar esquerdo (Ale).

Distância intercaninos (CSd-CSe) - Distância da ponta da cúspide do canino superior direito à ponta da cúspide do canino superior esquerdo.

Distância da distal intercaninos (dCSd-dCSe) Distância entre o ponto mais distal na porção média da coroa do canino direito ao ponto mais distal na porção média da coroa do canino esquerdo.

Largura da rima bucal (Abd-Abe) - Distância linear do ângulo da boca direito (Abd) ao ângulo da boca esquerdo (Abe).

Linha média dentária superior com linha média facial - Distância da linha média dentária superior e a linha média facial.

Centro do sorriso com linha média dentária superior - Distância do centro do sorriso até a linha média dentária superior.

Centro do sorriso com linha média facial Distância do centro do sorriso até a linha média facial.

Corredor bucal direito e esquerdo - Distância linear da face vestibular do último dente posterior que aparece no sorriso ao ângulo da boca dos lados esquerdo e direito. 


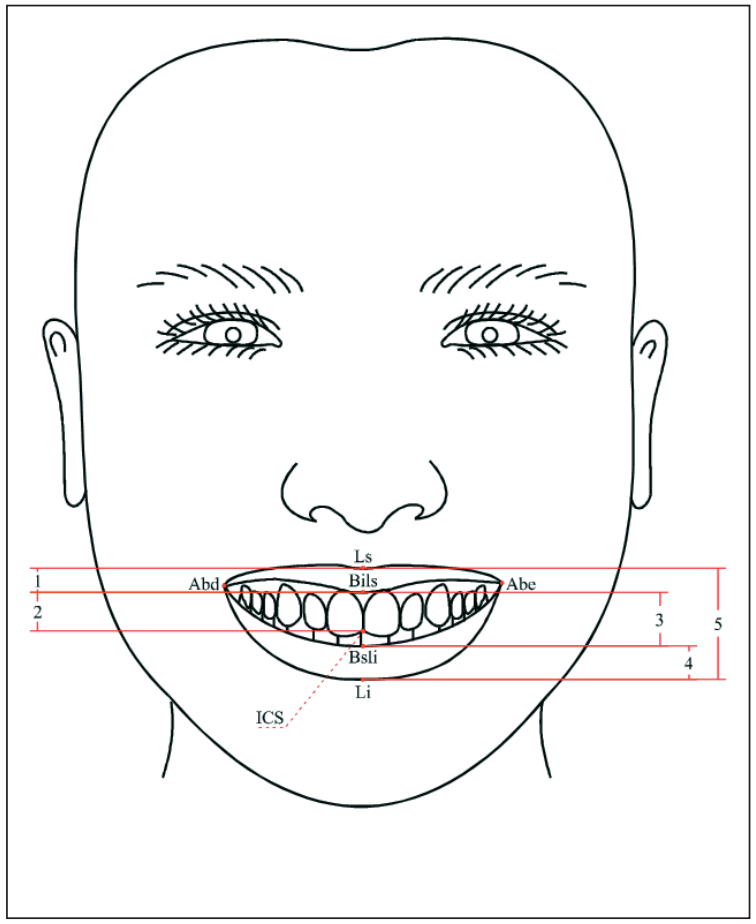

FIGURA 2 - Medidas fotométricas lineares.

01 - Extensão do vermelhão do lábio superior (Ls-Bils)

02 - Exposição dos incisivos centrais superiores (Bils-ICS)

03 - Espaço interlabial (Bils-Bsli)

04 - Extensão do vermelhão do lábio inferior (Bsli-Li)

05 - Altura do sorriso (Ls-Li)

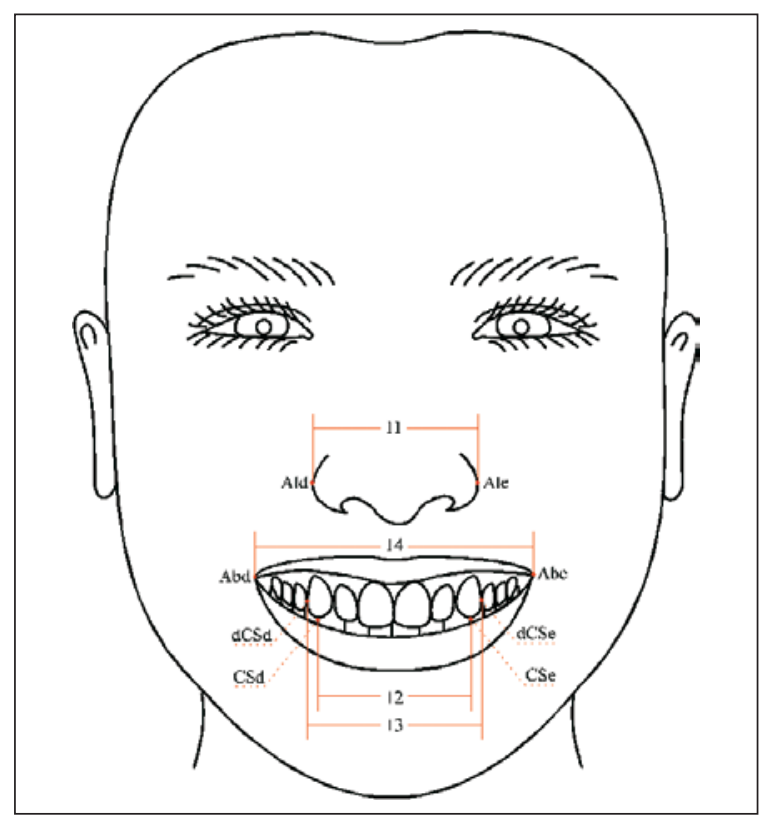

FIGURA 4 - Medidas fotométricas lineares.

11 - Largura do nariz (Ald-Ale)

12 - Distância intercaninos (CSd-CSe)

13 - Distância intercaninos distal (dCSd-dCSe)

14 - Largura da rima bucal (Abd-Abe)

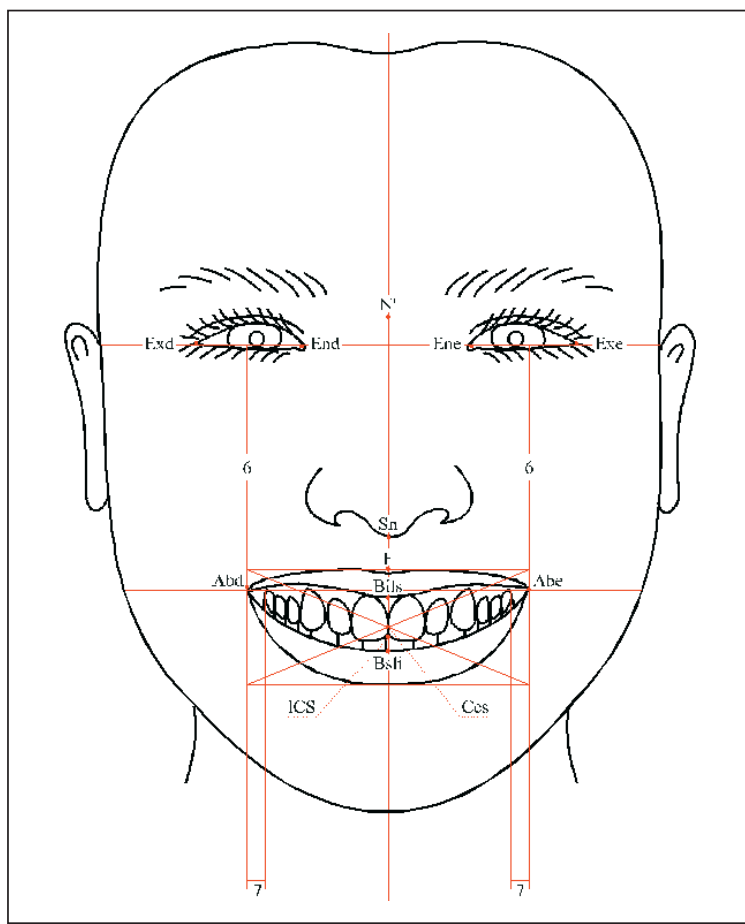

FIGURA 3 - Medidas fotométricas lineares.

06 - Inclinação da linha da comissura

07 - Corredor bucal direito e esquerdo

08 - Linha média dentária superior com linha média facial

09 - Centro do sorriso com linha média dentária superior

10 - Centro do sorriso com linha média facial

\section{Medidas fotométricas proporcionais}

Proporção Divina do sorriso ${ }^{35}$ - Proporção entre a largura do nariz (Ald-Ale) no sorriso e a distância intercaninos (CSd-CSe).

Proporção Divina do sorriso modificada Proporção entre a largura do nariz (Ald-Ale), durante o sorriso, e a distância entre o ponto mais distal do canino superior direito (dCSd) até a distal do canino superior esquerdo (dCSe).

Variáveis qualitativas (Fig. 5, 6).

Curvatura incisal superior em relação ao lábio inferior: a) paralela (em relação ao lábio inferior); b) reversa (canino mais baixo que os incisivos centrais superiores); c) reta (caninos estão na mesma altura que os incisivos centrais superiores) $)^{39}$.

Classificação do sorriso ${ }^{37}$ : a) sorriso canto da boca ou "Mona Lisa", os cantos da boca são puxados para cima e para fora, seguidos pela 


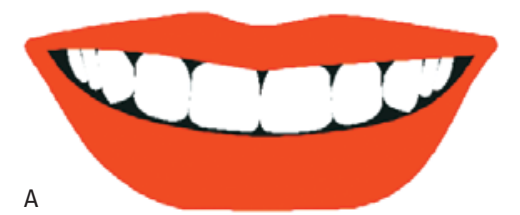

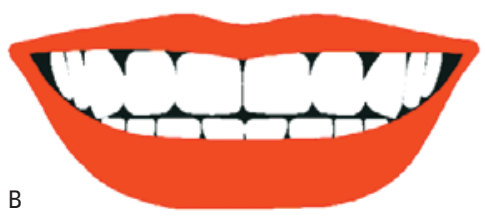

B

FIGURA 5 - Curvatura incisal superior em relação ao lábio inferior. A) paralela B) reversa C) reta
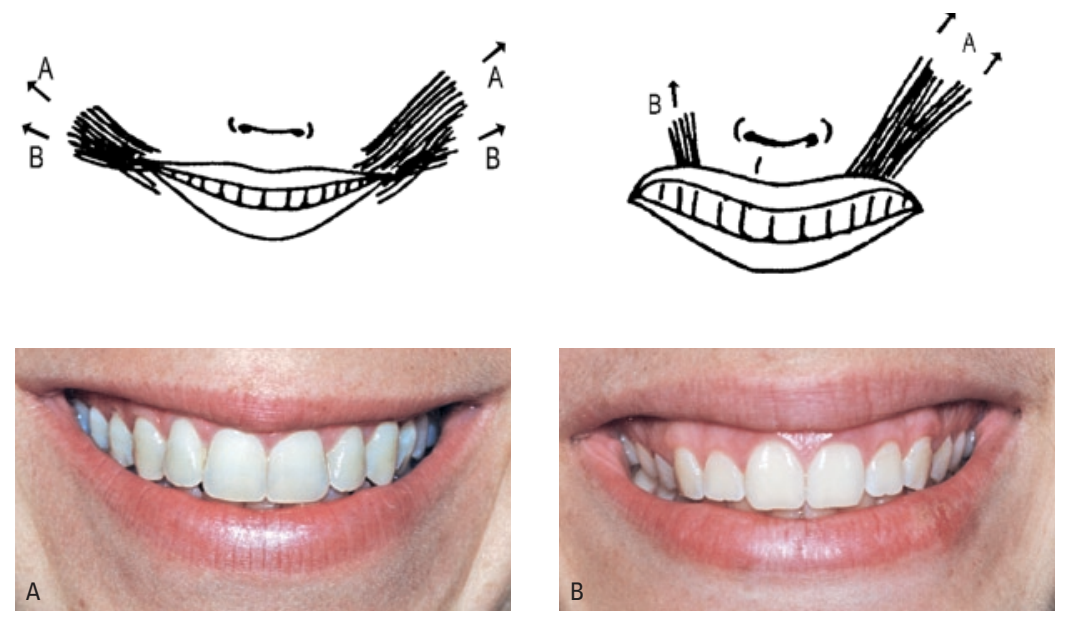

FIGURA 6 - Classificação do sorriso segundo Rubin ${ }^{37}$. A) Mona Lisa, B) canino, C) amplo.

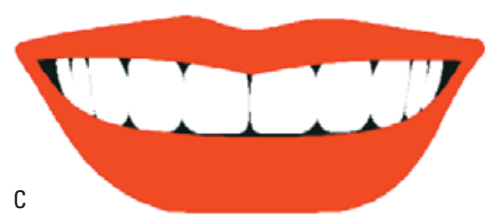

C
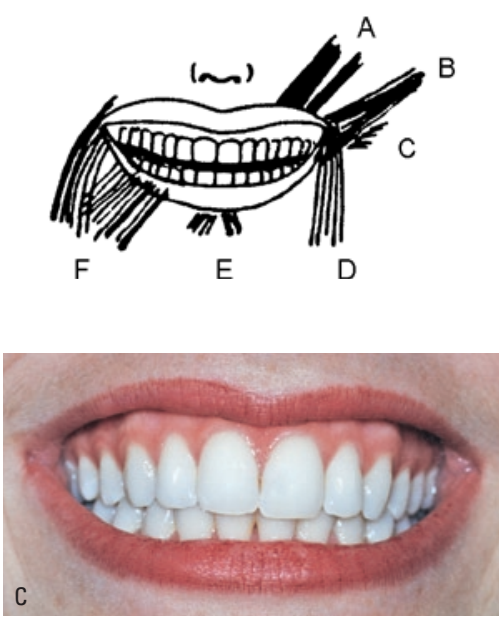

contração dos levantadores do lábio superior para mostrar os dentes superiores; b) sorriso "canino", os levantadores do lábio superior são dominantes e contraem, primeiramente, expondo os caninos superiores, os cantos da boca contraem, secundariamente, para puxar os lábios para cima e para fora; c) sorriso "amplo", em que todos os músculos contraem ao mesmo tempo: levantadores do lábio superior, levantadores dos ângulos da boca e abaixadores do lábio inferior, para mostrar todos os dentes superiores e inferiores.

Dentes que aparecem no sorriso - Dentes visíveis num sorriso natural, nos lados direito e esquerdo. 


\begin{tabular}{|c|c|c|c|c|c|c|c|}
\hline Variável & Média & Mediana & Mínimo & Máximo & $\begin{array}{l}\text { Desvio } \\
\text { Padrão }\end{array}$ & DP Erro & $\begin{array}{l}\text { Coef.de } \\
\text { Var }\end{array}$ \\
\hline $\begin{array}{l}\text { Largura do nariz } \\
\text { (Ald-Ale) }\end{array}$ & 21,69 & 21,50 & 19,00 & 25,00 & 1,34 & 0,224 & 6,19 \\
\hline $\begin{array}{l}\text { Dist. intercaninos } \\
\text { (CSd-CSe) }\end{array}$ & 18,96 & 19,00 & 16,00 & 21,00 & 1,09 & 0,194 & 5,74 \\
\hline $\begin{array}{l}\text { Dist. intercaninos } \\
\text { (dCSd-dCSe) }\end{array}$ & 20,83 & 21,00 & 18,50 & 23,50 & 1,08 & 0,224 & 5,20 \\
\hline $\begin{array}{l}\text { Largura rima bucal } \\
\text { (Abd-Abe) }\end{array}$ & 35,70 & 36,00 & 30,00 & 41,00 & 2,63 & 0,224 & 7,37 \\
\hline $\begin{array}{l}\text { Dist. entre a linha } \\
\text { média dent. sup. e } \\
\text { linha média facial }\end{array}$ & 0,19 & 0,00 & 0,00 & 1,00 & 0,33 & 0,112 & 177,99 \\
\hline $\begin{array}{l}\text { Dist. do centro do } \\
\text { sorriso à linha média } \\
\text { facial }\end{array}$ & 0,36 & 0,50 & 0,00 & 1,00 & 0,34 & 0,194 & 93,64 \\
\hline $\begin{array}{l}\text { Dist. do centro do } \\
\text { sorriso à linha média } \\
\text { dent. sup. }\end{array}$ & 0,39 & 0,50 & 0,00 & 1,50 & 0,43 & 0,158 & 111,22 \\
\hline
\end{tabular}

\begin{tabular}{|c|c|c|c|c|c|c|c|}
\hline Variável & Média & Mediana & Mínimo & Máximo & $\begin{array}{l}\text { Desvio } \\
\text { Padrão }\end{array}$ & Dp Erro & $\begin{array}{c}\text { Coef.de } \\
\text { Var }\end{array}$ \\
\hline $\begin{array}{l}\text { Largura do nariz } \\
\text { (Ald-Ale) }\end{array}$ & 21,69 & 21,50 & 19,00 & 25,00 & 1,34 & 0,224 & 6,19 \\
\hline $\begin{array}{l}\text { Dist. intercaninos } \\
\text { (CSd-CSe) }\end{array}$ & 18,96 & 19,00 & 16,00 & 21,00 & 1,09 & 0,194 & 5,74 \\
\hline $\begin{array}{l}\text { Proporção Divina do } \\
\text { sorriso }\end{array}$ & 1,156 & 1,14 & 1,00 & 1,34 & 0,10 & 0,102 & 6,47 \\
\hline $\begin{array}{l}\text { Largura do nariz } \\
\text { (Ald-Ale) }\end{array}$ & 21,69 & 21,50 & 19,00 & 25,00 & 1,34 & 0,224 & 6,19 \\
\hline $\begin{array}{l}\text { Dist. intercaninos } \\
\text { dCSd-dCSe }\end{array}$ & 20,83 & 21,00 & 18,50 & 23,50 & 1,08 & 0,224 & 5,20 \\
\hline $\begin{array}{l}\text { Proporção Divina do } \\
\text { sorriso modif. }\end{array}$ & 1,042 & 1,04 & 0,91 & 1,18 & 0,06 & 0,015 & 5,33 \\
\hline
\end{tabular}

Tabela 4 - Distribuição de freqüência e percentual da curvatura incisal superior em relaçào ao lábio inferior.

\begin{tabular}{|c|c|c|}
\hline Curvatura Incisal & Freqüência & Percentual \\
\hline Paralela & 33 & $82,5 \%$ \\
\hline Reversa & 00 & 00 \\
\hline Reta & 07 & $17,5 \%$ \\
\hline
\end{tabular}

Exposição gengival - distância da margem gengival do ICS à borda inferior do lábio superior (BiLs). Nos casos em que a margem gengival não aparece ela é considerada negativa.

\section{ANÁLISE ESTATÍSTICA}

Através da análise estatística, foram apresentadas medidas de posição e de dispersão para as variáveis contínuas das fotografias. Foram apresentadas as informações correspondentes à média amostral, mediana, valor mínimo observado, valor máximo observado, desvio padrão, erro padrão e coeficiente de variação.

Também foram apresentadas, para as variáveis contínuas, as informações correspondentes à média amostral, intervalo de 95\% de confiança para a média populacional e intervalo de $95 \%$ de confiança para as observações; o primeiro e o terceiro quartis e o valor do $\mathrm{p}$ resultante do teste de normalidade de Shapiro-Wilk.

A confiabilidade das medidas utilizadas neste trabalho foram avaliadas selecionando-se, aleatoriamente, as fotografias de 10 indivíduos entre os 40 , totalizando 20 fotografias: 10 fotografias em repouso e 10 durante o sorriso. Nessas fotografias, novos traçados e medições foram feitos. Para o cálculo do desvio padrão do erro foi utilizada a fórmula de Dahlberg ${ }^{19}$. Os erros acima de $1 \mathrm{~mm}$ para as medidas lineares e acima de $1,5^{\circ}$ para medidas angulares foram considerados como erros absolutos, altos e significantes.

\section{RESULTADOS}

Nas tabelas 1 a 3 são apresentadas, para as variáveis contínuas, as informações correspondentes à média amostral, mediana, valor mínimo observado, valor máximo observado, desvio padrão, desvio padrão do erro e coeficiente de variação. 


\begin{tabular}{|c|c|c|}
\hline Classif. do Sorriso & Freqüência & Percentual \\
\hline Canto da boca ("Mona Lisa") & 22 & $55 \%$ \\
\hline Canino & 09 & $22,5 \%$ \\
\hline Amplo & 09 & $22,5 \%$ \\
\hline
\end{tabular}

\begin{tabular}{|c|c|c|}
\hline Exposição Gengival & Freqüência & Percentual \\
\hline $0 \mathrm{~mm}$ & 13 & $32,5 \%$ \\
\hline $0,5 \mathrm{~mm}$ & 04 & $10 \%$ \\
\hline $1 \mathrm{~mm}$ & 04 & $10 \%$ \\
\hline Negativo & 19 & $47,5 \%$ \\
\hline
\end{tabular}

\section{DISCUSSÃO}

A análise da estética facial e do sorriso, apesar de ser subjetiva e difícil ${ }^{34}$, não é impossível de ser realizada.

O sorriso atraente é um complemento da beleza facial. Um sorriso simétrico, com dentes bem posicionados e alinhados no arco, uma exposição adequada dos dentes anteriores superiores, proporciona uma beleza ideal. Muitas pessoas consideradas belas, quando sorriem apresentam um sorriso assimétrico ou um sorriso gengival, perdendo o encanto inicial. A análise facial frontal, durante o sorriso, permite avaliar o alinhamento das linhas médias dentária e facial, a inclinação do sorriso, o centro do sorriso, o tipo de musculatura predominante, a curvatura incisal, o corredor bucal e a exposição gengival, os quais determinam o grau de atratividade de um sorriso.

Araújo e Tamaki ${ }^{1}$, analisando 20 mulheres entre 20 e 30 anos de idade, encontraram uma exposição dos incisivos de 9,9 mm. Rigsbee III, Sperry e Begole ${ }^{36}$, com uma amostra mista formada por mulheres tratadas ortodonticamente e não tratadas, obtiveram uma média de 10,4 mm. Peck, S., Peck, L. e Kataja ${ }^{32}$, em adolescentes norte-americanas de origem européia, obtiveram a média de $9,8 \mathrm{~mm}$. Para Arnett e Bergman ${ }^{2,3}$, durante o sorriso devem

\begin{tabular}{|c|c|c|}
\hline Dentes que aparecem no sorriso & Freqüência & Percentual \\
\hline $2^{\circ} \mathrm{PM}$ & 14 & $35 \%$ \\
\hline $1^{\circ} \mathrm{M}$ & 25 & $62,5 \%$ \\
\hline $2^{\circ} \mathrm{M}$ & 01 & $2,5 \%$ \\
\hline
\end{tabular}

ficar expostos de três quartos da coroa do incisivo superior até $2 \mathrm{~mm}$ de gengiva. Nanda e Ghosh ${ }^{31}$, estudando a face de mulheres jovens, leucodermas, obtiveram o valor médio de $8,93 \mathrm{~mm}$. A média de 9,3 mm, obtida nesta amostra, está muito próxima dos valores encontrados na literatura.

A extensão do vermelhão do lábio superior e inferior durante o sorriso, obtida em neste trabalho foi de 5,35 mm e 9,15 $\mathrm{mm}$, respectivamente. Comparando-se a extensão do vermelhão do lábio em repouso e durante o sorriso, no sorriso houve uma redução de $0,8 \mathrm{~mm}$ ou $11,91 \%$ do vermelhão do lábio superior e 1,58 mm ou 14,12\% de redução do vermelhão do lábio inferior.

Rigsbee III, Sperry e Begole ${ }^{36}$, obtiveram uma média de espaço interlabial de 12,34 mm. No atual trabalho obteve-se a média de $10,7 \mathrm{~mm}$, menor do que a encontrada por Rigsbee III, Sperry e Begole $^{36}$, provavelmente devido ao tipo de sorriso (canto de boca) predominante na amostra.

A altura do sorriso, medida entre o ponto Ls e Li, teve uma média de $25 \mathrm{~mm}$. Obteve-se um aumento de $8,13 \mathrm{~mm}$ ou $47,65 \%$ em relação à altura da boca em repouso. A altura da boca obteve o maior aumento percentual de todas as variáveis avaliadas.

$\mathrm{Na}$ amostra estudada, a média de inclinação da linha da comissura durante o sorriso, em relação à linha interexocanto foi de $0,75 \mathrm{~mm}$, menor do que durante o repouso, evidenciando uma simetria na contração da musculatura durante o sorriso.

Com relação à largura do nariz, durante o sorriso, Rigsbee III, Sperry e Begole ${ }^{36}$ observaram uma média de aumento de $4,26 \mathrm{~mm}$ ou $13,95 \% \mathrm{em}$ relação à largura do nariz em repouso. Na presente amostra, encontrou-se a média de 39,44 mm re- 
presentando um aumento de 4,62 $\mathrm{mm}$ ou 13,33\% em relação à largura do nariz em repouso. $\mathrm{O}$ aumento é equivalente ao encontrado por Rigsbee III, Sperry e Begole ${ }^{36}$.

Rigsbee III, Sperry e Begole ${ }^{36}$ obtiveram uma média de 37,91 mm para a distância intercaninos. Nesta pesquisa, a distância intercaninos na ponta da cúspide foi de $34,5 \mathrm{~mm}$ e a distância do ponto mais distal da porção média da coroa foi de $37,87 \mathrm{~mm}$. As mulheres constituintes da atual amostra apresentam uma distância intercaninos menor do que a encontrada por Rigsbee III, Sperry e Begole ${ }^{36}$. Essa medida permite avaliar se houve aumento da distância intercaninos com o tratamento ortodôntico.

Quanto à largura da rima bucal, obteve-se uma média de 64,9 mm. Houve um aumento de 15,79 mm ou $32,31 \%$ em relação à largura da boca em repouso. Rigsbee III, Sperry e Begole ${ }^{36}$, em sua amostra, obtiveram $66,22 \mathrm{~mm}$ durante o sorriso, 16,38 $\mathrm{mm}$ ou $33,02 \%$ maior do que em repouso, valores muito próximos aos encontrados neste trabalho.

Ocorreu um desvio médio da linha média dentária superior de $0,35 \mathrm{~mm}$ em relação à linha média facial. Este desvio não é significante clinicamente, e pode-se dizer que, na amostra estudada, houve um alinhamento entre a linha média facial e linha média dentária superior.

O centro do sorriso teve um desvio médio de $0,71 \mathrm{~mm}$ em relação à linha média dentária superior e $0,65 \mathrm{~mm}$ em relação à linha média facial. Um sorriso assimétrico pode levar ao desvio do centro do sorriso em relação às linhas médias. Idealmente, o centro do sorriso deveria coincidir com as linhas médias dentária superior e facial.

Para o valor do corredor bucal direito e esquerdo, a média foi, respectivamente, 5,65 mm e 5,61 $\mathrm{mm}$. Rigsbee III, Sperry e Begole ${ }^{36}$ obtiveram uma média, para o corredor bucal, de $0,57 \mathrm{~mm}$. Os valores obtidos diferem muito dos encontrados por Rigsbee III, Sperry e Begole ${ }^{36}$, provavelmente devido à metodologia utilizada. A definição de Frush e Fisher ${ }^{16}$ para o corredor bucal, foi utilizada na presente pesquisa. Essa medida serve para a indi- cação de expansão do arco superior em casos onde o corredor bucal é muito largo sugerindo, assim, uma possível atresia da maxila. Além disso, espaços negros no sorriso são antiestéticos. A largura do corredor bucal direito e esquerdo foram iguais revelando uma simetria durante o sorriso.

Com relação às Proporções Divinas do sorriso, largura do nariz durante o sorriso e a distância intercaninos, obteve-se, na média, uma proporção de 1:1,156 (nas medidas individuais tivemos apenas uma coincidência). Segundo Ricketts ${ }^{35}$, a proporção deve ser de 1:1. Por não encontrar a mesma proporção proposta por Ricketts ${ }^{35}$, utilizou-se outro ponto de referência na distal dos caninos superiores. A distância entre os pontos marcados na distal dos caninos foi denominada Proporção Divina do sorriso modificada. A proporção encontrada entre a largura do nariz durante o sorriso e a distância entre os pontos distais das coroas dos caninos superiores foi de 1:1,042, muito próxima da Proporção Divina preconizada na literatura. Nas medidas individuais dos 40 indivíduos ocorreram 12 coincidências.

Em relação à curvatura incisal, na amostra estudada, a maior incidência foi da curvatura incisal paralela ao lábio inferior $(82,5 \%)$, seguida pela curvatura incisal reta $(17,5 \%)$. Não foi encontrada nenhuma curvatura incisal reversa. Tjan e Miller ${ }^{39}$, analisando fotografias de mulheres entre 20 e 30 anos, observaram que $85,77 \%$ apresentavam curvatura incisal paralela, $13,56 \%$ curvatura reta e $0,6 \%$ curvatura reversa. Os resultados obtidos neste trabalho estão muito próximos dos encontrados pelos autores.

$\mathrm{Na}$ classificação do sorriso, segundo Rubin ${ }^{37}$, obteve-se 55\% das mulheres com sorriso do tipo canto de boca ou "Mona Lisa", 22,5\% com sorriso "canino" e 22,5\% com sorriso amplo. Rubin ${ }^{37}$, numa amostra de 100 mulheres, observou 67\% dos sorrisos sendo do tipo "Mona Lisa", 31\% do tipo "canino" e 2\%, "amplo". Neste estudo, a percentagem maior foi de sorriso do tipo canto de boca ou "Mona Lisa", concordando com os resultados de Rubin ${ }^{37}$. Quanto ao sorriso "canino" e "amplo", os resultados diferiram provavelmente devido à amostra utilizada. 
$\mathrm{Na}$ amostra pesquisada, a exposição gengival, durante o sorriso, variou entre um valor positivo de 1,8 $\mathrm{mm}$ até um valor negativo. A média percentual foi: $32,5 \%$ com exposição gengival igual a zero; $10 \%$ com 0,9 mm de exposição gengival; $10 \%$ com 1,8 mm e $47,5 \%$ com valor negativo. Rigsbee III, Sperry e Begole $^{36}$ encontraram uma média de $1,88 \mathrm{~mm}$ de exposição gengival. Peck, S., Peck, L. e Kataja ${ }^{32}$ obtiveram uma média de $0,7 \mathrm{~mm}$. Para Viazis ${ }^{40}$ durante o sorriso, os lábios devem expor de 0 a $2 \mathrm{~mm}$ de gengiva acima dos incisivos. Nanda e Ghosh ${ }^{31}$ encontraram uma média de $-1,31 \mathrm{~mm}$. Para Proffit ${ }^{34}$, durante o sorriso, somente uma pequena porção de gengiva deve ser exposta. Em $80 \%$ da amostra atual não foi encontrada exposição gengival. Este resultado foi, provavelmente, devido ao tipo de sorriso predominante em neste estudo (canto de boca ou "Mona Lisa"). A avaliação desse aspecto do sorriso é importante nos casos cirúrgicos de impacção maxilar, auxiliando na determinação de quantos milímetros a maxila deveria ser intruída superiormente.

Com relação à confiabilidade das medidas empregadas nesta análise facial do sorriso, nenhuma variável apresentou um desvio padrão do erro superior a $1 \mathrm{~mm}$ para as variáveis lineares, e superior a $1,5^{\circ}$ para as angulares. Portanto, as medidas utili-

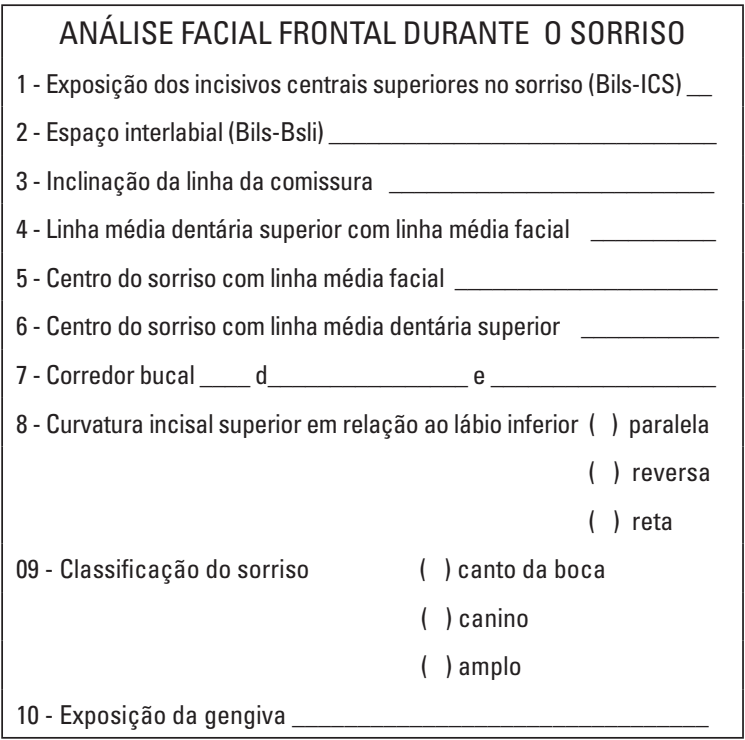

QUADRO 1 - Análise facial durante sorriso. zadas em nesta análise são confiáveis.

Além disto, com a padronização da metodologia, pode-se fazer comparações de trabalhos com amostras de mesma origem étnica e de origens étnicas diferentes, o que auxiliaria muito o campo de pesquisa da análise facial.

A análise facial do sorriso em fotografias frontais padronizadas pode ser utilizada como auxiliar importante no diagnóstico e planejamento do tratamento ortodôntico e cirúrgico, permitindo uma avaliação quantitativa antes e durante o tratamento, bem como apresentando-se como um método confiável para comparações entre o pré e o póstratamento. Entretanto, é importante ressaltar que ela não dispensa a avaliação clínica e radiográfica da face do paciente. Assim, propõem-se uma análise facial frontal durante o sorriso em fotografias para uso clínico, ilustrada no quadro 1.

\section{CONCLUSÕES}

Após a exposição e discussão dos resultados, parece lícito concluir que:

1) Os padrões médios de normalidade para as variáveis da análise da fotografia frontal durante $o$ sorriso da amostra estudada aparecem no quadro 2.

2) Nem todas as medidas utilizadas neste trabalho se assemelham às encontradas na literatura.

3) A variável largura do nariz, durante o sorriso, teve uma média de aumento de 4,62 mm, $13,33 \%$ maior que em repouso. A largura da boca aumentou $15,79 \mathrm{~mm}, 32,31 \%$ maior que em repouso. A altura da boca obteve o maior aumento percentual de todas as variáveis avaliadas: $47,64 \%$ ou $8,13 \mathrm{~mm}$ de aumento durante o sorriso. A altura do vermelhão do lábio superior e inferior tiveram uma redução de $11,91 \%$ e $14,14 \%$, respectivamente, durante o sorriso. O lábio superior teve uma média de redução de $0,8 \mathrm{~mm}$ e o inferior $1,58 \mathrm{~mm}$ durante o sorriso.

4) O sorriso canto de boca ou "Mona Lisa" foi predominante (55\%). Quanto a curvatura incisal, esta foi paralela em relação ao lábio inferior, em $82 \%$ da amostra. 
5) As Proporções Divinas propostas por Ricketts $^{35}$ não são válidas para a amostra estudada.

6) Todas a variáveis mostraram-se confiáveis após a repetição das medições.
7) A proposta de uma análise facial tem se mostrado bastante útil nos procedimentos de diagnóstico e planejamento do tratamento ortodôntico e cirúrgico.

\begin{tabular}{|c|c|c|}
\hline VARIÁVEL & MÉDIA OBTIDA NA & MEDIDA "REAL" \\
\hline FOTOGRAFIA & \\
\hline Ext. do vermelhão do lábio sup. (Ls-Bils) & 2,94 & 5,35 \\
\hline Exp. dos incisivos cent. sup. (Bils-ICS) & 5,11 & 9,30 \\
\hline Espaço interlabial (Bils-Bsli) & 5,88 & 10,70 \\
\hline Ext. do vermelhão do lábio inf. (Bsli-Li) & 5,03 & 9,15 \\
\hline Altura do sorriso (Ls-Li) & 13,75 & 25,00 \\
\hline Inclinação da linha da comissura & 0,41 & 0,75 \\
\hline Largura do nariz (Ald-Ale) & 21,69 & 39,44 \\
\hline Dist. intercaninos (CSd-CSe) & 18,96 & 34,5 \\
\hline Dist. da distal intercaninos (dCSd-dCSe) & 20,83 & 37,87 \\
\hline Largura da rima bucal (Abd-Abe) & 35,70 & 64,90 \\
\hline Linha média dentária superior com linha média & 0,19 & 0,35 \\
\hline facial & & \\
\hline Centro do sorriso com linha média dentária & 0,39 & 0,71 \\
\hline superior & & 0,65 \\
\hline Centro do sorriso com linha média facial & 0,36 & 5,65 \\
\hline Corredor bucal direito & 3,11 & 5,62 \\
\hline Corredor bucal esquerdo & 3,09 & \\
\hline
\end{tabular}

QUADRO 2 - Fotografia frontal durante 0 sorriso.

\title{
Facial frontal analysis in rest position and maximun smile in standardized photoghaphs
}

\begin{abstract}
This is the second part of the frontal facial analysis using standardized photographs. The sample consisted of 40 white female with a mean age of 22 years old, pleasant face, normal occlusion, and had not been previously submitted to orthodontic treatment nor plastic surgery. Colorful standardized 10x15 cm photographs were obtained with the patients in their natural head position. Two pictures were taken for each subject, one in maximum smile and another in rest position. Linear, angular and proportional measurements were used. Tests of normality, descriptive statistics, and error standard deviations were executed. The results showed that some of the measurements used in this study have values similar to that found in the literature and some are very different. All of the measurements were reliable after their repetition. And after this study, it is suggested a frontal facial analysis to be used regularly in the diagnosis and orthodontic treatment planning.
\end{abstract}

Key words: Facial frontal analysis. Orthodontics. Facial aesthetics. Smile. 


\section{REFERÊNCIAS}

1. ARAÚJO, C. H.; TAMAKI, T. Posição labial em repouso e sorriso e sua relação com os incisivos centrais superiores. R Odontol Univ São Paulo, São Paulo, v. 1, n. 2, p. 28-34, abr./jun. 1987.

2. ARNETT, W. G.; BERGMAN, R. T. Facial keys to orthodontic diagnosis and treatment planning - part I. Am J Orthod Dentofacial Orthop, St. Louis, v. 103, no. 4, p. 299-312, Apr. 1993.

3. ARNETT, W. G.; BERGMAN, R. T. Facial keys to orthodontic diagnosis and treatment planning - part II. Am J Orthod Dentofacial Orthop, St. Louis, v.103, no. 5, p. 395-441, May 1993.

4. BISHARA, S. E.; JORGENSEN, G. J.; JAKOBSEN, J. R. Changes in facial dimensions assessed from lateral and frontal photographs. Part I - Metodology. Am J Orthod Dentofacial Orthop, St. Louis, v.108, no. 4, p. 389-393, Oct. 1995.

5. BISHARA, S. E.; JORGENSEN, G. J.; JAKOBSEN, J. R. Changes in facial dimensions assessed from lateral and frontal photographs. Part II - Results and conclusions. Am J Orthod Dentofacial Orthop, St. Louis, v. 108, no. 5, p. 489-499, Nov. 1995.

6. CHIU, C. S. W.; CLARK, R. K. F. Reproducibility of natural head position. J Dent Hong Kong, [S. I.], v.19, p.130-131, Jan.1991.

7. COLOMBO, V. L.; et al. Análise facial frontal em repouso e durante o sorriso em fotografias padronizadas. Parte I. Avaliação em repouso. R Dental Press Ortodon Ortop Facial, Maringá. No prelo.

8. COOKE, M. S.; WEI, S. H. Y. The reproducibility of natural head posture: a methodological study. Am J Orthod Dentofacial Orthop, St. Louis, v. 93, no. 4, p. 280-288, Apr. 1988.

9. CUMMINS, D. M.; BISHARA, S. E.; JAKOBSEN, J. R. A computer assisted photogrammetric analysis of soft tissue changes after orthodontic treatment. Part II. Results. Am J Orthod Dentofacial Orthop, St. Louis, v.108, no.1, p. 38-47, July 1995.

10. EL-MANGOURY, N. H.; MOSTAFA, Y. A. Faciometrics: A new syntax for facial feature analysis. Int J Adult Orthod Orthognath Surg, Lombard, v.11, no.1, p.17-29, 1996.

11. ELLENBOGEN, R.; SWARA, N. The Improvement of the gummy smile using the implant spacer technique. Ann Plastic Surg, Los Angeles, v.12, no.1, p.16-24, Jan. 1984.

12. EPKER, B. N. Adjuntctive esthetic surgery in the orthognathic surgery patient. In: McNAMARA Jr., J. A. Esthetic and the Treatment of Facial Form. Ann Arbor: University of Michigan, Center for Human Growth and development, 1992. p. 18-35. Craniofacial Growth Series, v. 28.

13. FARKAS, L. G.; BRYSON, W.; KLOTZ, J. Is photogrammetry of the face reliable? Plast Reconstr Surg, Toronto, v. 66, no. 3, Sept. 1980.

14. FERRARIO, V. F. et al. Craniofacial morphometry by photographic evaluations. Am J Orthod Dentofacial Orthop, St. Louis, v.103, no. 4, p. 327-337, Apr. 1993.

15. FRICKER, J. P. Standardized facial photography. Austr Orthod J, Sydney, v. 7, p.168-173, Oct.1982.

16. FRUSH, J. P.; FISHER, R. D. The Dynesthetic interpretation of the dentogenic concept. J Prost Dent, St. Louis, v. 8, no. 4, p. 558-581, July 1958.

17. GIBSON, R. M. Smiling and facial exercise. Dent Clin Am, Philadelphia, v. 33, no. 2, p. 139-144, Apr. 1989.

18. GIDDON, D. B. Aplicações ortodônticas de estudos psicológicos e perceptuais da estética facial. In: SADOWSKY, P. L. et al. Atualidades em Ortodontia. São Paulo: Premier, 1997. p. 79-88.

19. HOUSTON, W. J. B. The analysis of errors in orthodontic measurements. Am J Orthod, St. Louis, v. 83, no. 5, p. 382-390, May 1983.

20. HULSEY, C. M. An evaluation of lip-teeth relationships present in the smile. Am J Orthod, St. Louis, v. 57, no. 2, p. 133-144, Feb. 1970.
21. JANSEN, E. K. A balanced smile: a must important treatment objective. Am J Orthod, St. Louis, v. 72, no. 4, p. 359-372, Oct. 1977.

22. LITTON, C.; FOURNIER, P. Simple surgical correction of the gummy smile. Plast Reconstr Surg, Baltimore, v. 63, no. 3, p. 372-373, Mar.1979.

23. LOMBARDI, R. E. The principles of visual perception and their clinical application to denture esthetic. J Prost Dent, St. Louis, v. 29, no. 4, p. 358-382, Apr. 1973.

24. MACKLEY, R. J. Development of balance and harmony of the smile. Func Orthod, Winchester, v.10, no. 3, p. 5-21, May/June 1993.

25. MATTHEWS, T. G. The anatomy of a smile. J Prost Dent, St. Louis, v. 39, no. 2, p.128-134, Feb. 1978

26. McNAMARA Jr., J. A.; BRUST, E. W.; RIOLO, M. L. Soft tissue evaluation of individuals with an ideal occlusion and a well-balanced face. In: McNAMARA Jr., J. A. Esthetic and the treatment of facial form. Ann Arbor: University of Michigan. Center for Human Growth and Development, 1992. p. 185-212. Craniofacial Growth Series, v. 28.

27. MISKINYAR, S. A. C. A new method for correcting gummy smile. Plast Reconstr Surg, Baltimore, v. 72, no. 3, p. 397-400, Sept. 1983.

28. MOORREES, C. F. A. Natural head position: a revival. Am J Orthod Dentofacial Orthop, St. Louis, v. 105, no. 5, p. 512-513, May 1994.

29. MORO, A. et al. Análise de alguns fatores estéticos do sorriso. Porto Alegre: [s.n.], 1994. Apresentado no $10^{\circ}$ Congresso Odontológico Riograndense.

30. MOSKOWITZ, M. E.; NAYYAR, A. Determinants of dental esthetics: A rationale for smile analysis and treatment. Compendium, Newtown, v.16, no.12, p. 13-22, Dec.1995.

31. NANDA, R. S.; GHOSH, J. Harmonia entre os tecidos moles da face e o crescimento no tratamento ortodôntico. In: SADOWSKY, P. L. et al. Atualidades em Ortodontia. São Paulo: Premier, 1997. p. 65-78.

32. PECK, S.; PECK, L.; KATAJA, M. Some vertical lineaments of lip position. Am J Orthod Dentofacial Orthop, St. Louis, v. 101, no. 6, p. 519-524, June 1992.

33. PHILLIPS, C. et al. Photocephalometry: Errors of projection and landmark location. Am J Orthod, St. Louis, v. 86, no. 3, p. 233243, Sept. 1984.

34. PROFFIT, W. The search for truth. In: PROFFIT, W.; WHITE Jr., R. P. Surgicalorthodontic treatment. St. Louis: C. V. Mosby, 1991. p. 96-141.

35. RICKETTS, R. M. The biologic significance of the divine proportion and Fibonacci series. Am J Orthod, St. Louis, v. 81, no. 5, p. 351-370, May 1982.

36. RIGSBEE III, O. H.; SPERRY, T. P.; BEGOLE, E. A. The influence of facial animation on smile characteristics. Int $\mathbf{J}$ Adult Orthod Orthognath Surg, Lombard, v. 3, no. 4, p. 233-239, 1988.

37. RUBIN, L. R. The Anatomy of a smile: its importance in the treatment of facial paralysis. Plast Reconstr Surg, Baltimore, v. 53, no. 4, p. 384-387, Apr. 1974.

38. STRAUSS, R. A. et al. Variability of facial photographs for use in treatment planning for orthodontics and orthognathic surgery. Int J Adult Orthod Orthognath Surg, Lombard, v.12, no. 3, p. 197-203, 1997.

39. TJAN, A. H. L.; MILLER, G. D. Some esthetic factors in a smile. J Prost Dent, St. Louis, v. 51, no.1, p. 24-28. Jan 1984

40 VIAZIS, A. D. Avaliação do tecido mole: atlas de Ortodontia: princípios e aplicações clínicas, 1996. p. 49-57.

41 ZACHRISSON, Bjorn U. Esthetic factors involved in anterior tooth display and the smile: vertical dimension. J Clin Orthod, Boulder, v. 23, no. 7, p. 432-445, July 1998.

Endereço para correspondência

Alexandre Moro - Av. Silva Jardim, 2675 Água Verde

Curitiba-PR. Brasil.

CEP: 80240-020

E-mail: amoro@bio.ufpr.br 